\title{
Downregulation of microRNA-17-5p improves cardiac function after myocardial infarction via attenuation of apoptosis in endothelial cells
}

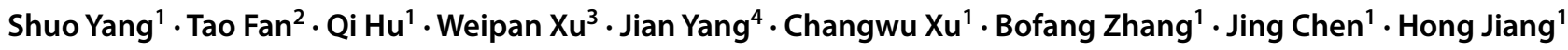

Received: 10 July 2017 / Accepted: 14 February 2018 / Published online: 13 March 2018

(c) The Author(s) 2018

\begin{abstract}
MicroRNA-17-5p (miR-17-5p) was indicated to suppress the formation of blood vessels, which is associated with cardiac function after myocardial infarction. In this study, the relationship between miR-17-5p and cardiac function was researched. Human umbilical vein endothelial cells were infected with adenoviruses. Apoptosis was determined by Annexin V-7AAD/ PI. Real-time RT-PCR was used to evaluate miR-17-5p and ERK levels. Western blotting was used to determine the levels of ERK, the anti-apoptosis protein bcl-2 and apoptosis proteins, including bax, caspase 3, and caspase 9. An in vivo acute myocardial infarction (AMI) model was established in SD male rats. Heart function was evaluated by echocardiography prior to inducing AMI and after 7 and 28 days later. The heart was removed to perform histological examination, real-time RT-PCR, and western blotting, as described above. The result indicated that the ERK pathway was activated by miR-17-5p downregulation and an increase in the level of the anti-apoptosis protein bcl-2; however, the levels of apoptosis proteins (bax/ caspase 3/caspase 9) were decreased. The results were completely reversed when miR-17-5p was up-regulated. At 7 and 28 days after the induction of AMI, in the miR-17-5p inhibition group, the infarction areas and collagen fibers were decreased, apoptosis in cardiac tissues was inhibited, and the endothelial growth process was promoted. Therefore, MiR-17-5p silencing protects heart function after AMI through decreasing the rate of apoptosis and repairing vascular injury.
\end{abstract}

Keywords MicroRNA-17-5p $\cdot$ Endothelial cells $\cdot$ Apoptosis $\cdot$ Myocardial infarction

\section{Introduction}

Myocardial infarction is one of the leading causes of death globally and is mainly characterized by vascular endothelial injury due to apoptosis, cardiac fibrosis, inflammation, and pathological remodeling (Du et al. 2014). Though

Communicated by S. Hohmann.

Hong Jiang

phy_hongj@163.com

1 Department of Cardiology, Renmin Hospital of Wuhan University, Cardiovascular Research Institute of Wuhan University, 238 JieFang Road, Wuhan 430060, China

2 Department of Thoracic Surgery, Remin Hospital of Wuhan University, Wuhan, China

3 Department of Cardiology, Huangshi Central Hospital, Huangshi, China

4 Department of Cardiology, Yichang Central People's Hospital, Yichang, China precautions and medical treatments for myocardial infarction have improved, a considerable number of deaths due to myocardial infarction occur every year (Zhang et al. 2012). Heart failure after myocardial infarction (MI) is closely associated with microcirculation (Al Suwaidi et al. 2000; Joner et al. 2006; Farb and Boam 2007; Dangas et al. 2010). Studies have confirmed that the common pathological physiological basis of myocardial infarction is delayed repair of the damaged vascular endothelium (Finn et al. 2007; Inoue et al. 2011). It is highly important to completely restore endothelial function soon after myocardial infarction.

MicroRNA is a newly discovered class of small nonprotein-coding mRNAs, which have rapidly became known as critical regulators of gene expression (Krol et al. 2010). Mature microRNAs negatively regulate gene expression through two mechanisms: they prevent protein synthesis at the translational level and cause degradation of the mRNA of the target gene (van Rooij 2011). Every microRNA can control the expression of multiple genes simultaneously, which makes the translation of traditional single locus 
control to multiple site intervention possible (van Rooij 2011). Cell-specific expression is another major characteristic of microRNAs (van Rooij 2011). Through post-transcriptional regulation, microRNAs define the proteomes of cells by regulating gene expression, and their regulatory activities are involved in various cellular functions and generate specific biological phenotypes (Norbury 2010). Recently, many miRNAs have been confirmed to be involved in the regulation of the pathological and physiological processes of heart disease (Ren et al. 2009; Wang et al. 2010; Pan et al. 2012).

Researchers have shown that microRNA-17 (miR-17) is highly expressed in endothelial cells (EC) (Bonauer et al. 2009) and has low expression in vascular smooth muscle cells (VSMC). Researchers have also found that miR-17 exerts an anti-angiogenic function; therefore, inhibiting its expression can improve the density of EC angiogenesis in vitro (Doebele et al. 2010; Yu et al. 2010). Other studies have indicated that in the process of rat carotid artery injury and repair, the expression of miR-17 rises continuously, while the expression of miR-18a and miR-19a, which are known to promote angiogenesis, show no obvious changes (Ji et al. 2007). Here, we hypothesized that downregulation of miR-17 would enhance cardiac function after MI by improving endothelial function and increasing the density of new blood vessels.

\section{Materials and methods}

\section{Cell culture and adenovirus infection}

Human umbilical vein endothelial cells (HUVECs) were purchased from Sciencell Company (Cat. No. 8000) and cultured with endothelial cell medium (ECM) (Sciencell Cat. No. 1001) containing 5\% fetal bovine serum (FBS), $1 \%$ endothelial cell growth supplement (ECGS), and 1\% penicillin/streptomycin solution (P/S). All HUVECs were used for experiments between the first and fifth passages. Recombinant adenoviruses containing pre-miR-17 or Antagomir-17 for miR-17-5p overexpression (Ad-PremiR-17) and miR-17-5p silencing (Ad-Antagomir-17), respectively, were prepared. Ad-NC-Pre-miR-17, which contains the same gene sequence as Pre-miR-17, but does not affect the expression of miR-17-5p, was used as the control adenovirus for Ad-Pre-miR-17. Ad-NC-Antagomir-17, which contains the same gene sequence as Antagomir-17, but does not affect the expression of miR-17-5p, was used as the control adenovirus for Ad-Antagomir-17. For adenovirus infection, the above-mentioned HUVECs were incubated with Ad-Pre-miR-17/Ad-NC-Pre-miR-17 or Ad-Antagomir-17/Ad-NC-Antagomir-17 at a multiplicity of infection (MOI) of 30 in serum-free media for approximately $4 \mathrm{~h}$. Then, the media were removed, and the cells were incubated with complete medium for the next $72 \mathrm{~h}$.

\section{Apoptosis analysis}

Cell apoptosis was measured in an Annexin V-7AAD/propidium iodide (PI) assay. HUVECs infected with the recombinant adenoviruses mentioned above were divided into three parts and seeded in 60 -mm plates $\left(1 \times 10^{5}\right)$. After synchronization in ECM with $0.5 \%$ FBS for $24 \mathrm{~h}$, one part was cultivated in ECM containing 5\% FBS for $24 \mathrm{~h}$, and the second part underwent apoptosis in ECM without FBS for $24 \mathrm{~h}$, and the third part treated with ERK inhibitor PD098059 at a concentration of $10 \mu \mathrm{M}$ in ECM without FBS for $24 \mathrm{~h}$. Cells were collected and re-suspended in $1 \times$ binding buffer. Apoptotic cells were measured with an Annexin V-7AAD apoptosis detection kit (BD Biosciences, USA) according to the manufacturer's protocol. Five microliters of Annexin $\mathrm{V}-7 \mathrm{AAD}$ and $5 \mu \mathrm{l}$ of PI were added to the re-suspended cells, and the cells were incubated for $15 \mathrm{~min}$ at room temperature in the dark. Apoptosis was measured using a BD accuriC6 flow cytometer, and the data were processed using FlowJo (FlowJo, Ashland, OR, USA) software.

\section{Real-time RT-PCR}

Total RNA was extracted from cells or hearts with Trizol reagent (Invitrogen), then reversely transcribed to cDNA using Quantscript RT kit (Tiangen, Beijing, China). U6 and mRNAs were converted using random hexamers, while miR17 was reversely transcribed using gene-specific primer. qPCR was performed in triplicates using SYBR Premix Ex TaqTM II (TaKaRa, Japan) on Step-one plus real-time PCR system (Applied Biosystems, Foster City, CA, USA). GAPDH (for mRNAs) and U6 (for microRNAs) were used as internal controls for normalization and gene expression level was calculated using 2- $\Delta \Delta \mathrm{Ct}$ method. The primer sequences were as follows:

miR-17-5p Forward primer: 5'GGGGCAAAGTGCTTA CAGTG3'

Reverse primer: 5'GTGCGTGTCGTGGAGTCG3'

U6 Forward primer: 5'GCTTCGGCAGCACATATACTA AAAT3'

Reverse primer: 5'CGCTTCACGAATTTGCGTGTCAT3'

ERK Forward primer: 5'GAGGTTGACCACGGTGGA AT3'

Reverse primer: 5' TTTGGTTTCCCACGGCTTCT3'

GAPDH Forward primer: 5'GGGAAACTGTGGCGT GAT3'

Reverse primer: 5'GAGTGGGTGTCGCTGTTGA3' 


\section{Western blotting}

For whole-cell protein extraction, HUVECs under apoptosis induction were washed three times with cold PBS and subsequently lysed in cold RIPA lysis buffer $(20 \mathrm{mM}$ Tris $\mathrm{Cl}$, $\mathrm{pH} 7.4 / 137 \mathrm{mM}, \mathrm{NaCl} / 2 \mathrm{mM}$ EDTA/1\% Triton/10\% glycerol/25 mM $\beta$-glycerol phosphate/1 mM PMSF, and protease inhibitor mixture). Cell or heart lysis was performed on ice for $30 \mathrm{~min}$, and clear protein extracts were obtained by centrifugation at $12,000 \times g$ for $10 \mathrm{~min}$. Protein concentration was determined with the bicinchoninic acid protein assay (Bipec). Proteins were separated by SDS-polyacrylamide gel electrophoresis and were transferred to PVDF membranes. For immunoblotting, PVDF membranes were blocked with $5 \%$ milk and probed with antibodies against total ERK and phospho-ERK, bax, bcl-2, caspase 3, caspase 9 or GAPDH (all from Abcam) overnight at $4{ }^{\circ} \mathrm{C}$. After washing the membranes with TBST three times (10 min each time), they were incubated with peroxidase-conjugated secondary antibodies (BOSTER) for $2 \mathrm{~h}$ at room temperature. The proteins were detected using the ECL Plus detection kit (Bipec).

\section{SD rat AMI model}

All animals used in this study were provided and cared for by the Animal Center of Renmin Hospital of Wuhan University. The experimental procedures and animal care were approved by the Animal Care and Use Committee of Wuhan University. All animals were given a conventional diet until they were sacrificed. All of the animal protocols complied strictly with the Institutional Animal Care and Use Committee guidelines. The procedure for inducing myocardial infarction (MI) in rats was described previously. Briefly, male Sprague-Dawley (SD) rats (200-250 g) were anesthetized with pentobarbital $(30 \mathrm{mg} / \mathrm{kg}$ intraperitoneal injection). The adenoviruses $\left(5 \times 10^{9} / 100 \mu \mathrm{L}\right)$ mentioned above were injected into the apex of the heart after the left anterior descending branch (LAD) was ligatured. The rats were euthanized at 7 or 28 days after MI, and the tissues were harvested for specific protocols. The rats were divided into four groups with five rats in each group: group 1: AdNC-Antagomir-17 for 7 days, group 2: Ad-Antagomir-17 for 7 days, group 3: Ad-NC-Antagomir-17 for 28 days, and group 4: Ad-Antagomir-17 for 28 days. The sham groups were exposed to the surgery without AMI and continued to be fed for 7 or 28 days. Cardiac function was evaluated by echocardiography before surgery and was re-evaluated at 7 or 28 days after MI. For histology, the tissues were perfusion-fixed with buffered formalin phosphate, and the hearts were harvested and processed. For real-time RT-PCR and western blot, the tissues were freshly removed and snapfrozen, as described above.

\section{Morphometric analysis}

At 7 or 28 days after the operation, the hearts were harvested, fixed in $4 \%$ paraformaldehyde, and then embedded in paraffin. For morphologic analysis, cardiac tissue at the infarction border area was removed. The infarction areas were determined by HE staining. Masson staining was used to examine collagen fiber areas. Apoptosis of cardiac tissue was determined by TUNEL staining; data are presented as percentages of total cells that are positive for apoptosis within a given area. CD31 staining was used to evaluate microcirculation formation; the perimeters of the areas positive for CD31 were measured, and the percentages of the total perimeters were also calculated.

\section{Statistical analysis}

Data are presented as means \pm SEM. All values were analyzed using Student's $t$ test for comparisons between two groups. A $p$ value of $<0.05$ was considered statistically significant.

\section{Results}

\section{Inhibition of miR-17-5p attenuates HUVEC apoptosis induced by culturing in ECM without FBS}

After infection with adenoviruses, the level of miR-17-5p in the HUVECs was determined by real-time RT-PCR to evaluate the transfection efficiency. The miR-17-5p level was downregulated by $23 \%$ in the Ad-Antagomir- 17 group and was up-regulated by 2.7-fold in the Ad-Pre-miR-17 group. Mir-17-5p silencing inhibited apoptosis in apoptosisinduced HUVECs. Inversely, up-regulation of miR-17-5p promoted apoptosis in apoptosis-induced HUVECs. However, in HUVECs cultured with ECM containing 5\% FBS, the apoptosis ratios were low in both the miR-17-5p overexpression and miR-17-5p silencing HUVECs. To further assess whether apoptosis inhibition by miR-17-5p depends on the expression of ERK in HUVECs, the inhibitory effect of the ERK inhibitor PD098059 was evaluated. Treatment with PD98059 did not inhibit HUVEC apoptosis after miR17-5p silencing in vitro (Fig. 1).

\section{Mir-17-5p silencing stimulates ERK-signaling pathway}

To further explore the mechanism of miR-17-5p on apoptosis, several key apoptosis pathways were examined. In apoptosis-induced HUVECs, the ERK pathway was stimulated by miR-17-5p silencing, as indicated by increased mRNA level of ERK and protein levels of ERK and phosphorylated 
Fig. 1 MicroRNA-17-5p inhibition suppressed HUVEC apoptosis. a HUVECs were infected with adenovirus for $4 \mathrm{~h}$ and cultured with ECM containing 5\% FBS for $72 \mathrm{~h}$. Total mRNA was extracted, and the miR-17-5p level was detected by real-time RT-PCR $(n=3)$. b HUVECs infected with adenoviruses were divided into two parts; one part underwent apoptosis induction via incubation with ECM lacking FBS for $24 \mathrm{~h}$, and the other part was cultured with ECM containing $5 \%$ FBS. Quantification of cellular apoptosis was performed with an Annexin V-7AAD/PI assay. c Apoptosis of HUVECs pretreated with the ERK inhibitor PD98059 $(10 \mu \mathrm{M})$ was evaluated by an Annexin V-7AAD/PI assay $\left({ }^{*} p<0.05\right.$ vs. Ad-NC-Pre-miR- $17,{ }^{\#} p<0.05$ vs. Ad-NC-Antagomir-17, $n=3$ )

ERK (P-ERK). ERK activity was evaluated by calculating P-ERK/ERK (Fig. 2).

\section{Mir-17-5p silencing inhibits apoptosis through the ERK signaling pathway}

To further investigate the effect of miR-17-5p on apoptosis via ERK pathway, several key downstream proteins were examined. In apoptosis-induced HUVECs, the protein level of the anti-apoptosis protein bcl-2 increased, and the levels of apoptosis proteins, including bax, caspase 9, and caspase 3, were reduced by miR-17-5p silencing; the opposite effect was observed with miR-17-5p overexpression (Fig. 3).

\section{Inhibition of miR-17-5p inhibits apoptosis of rat heart after AMI via the ERK pathway}

As shown in Figs. 4 and 5, miR-17-5p levels were decreased by 19.7 and $41.2 \%$, respectively, in hearts from rats in the Ad-Antagomir-17 group and the Ad-NC-Antagomir-17 group at 7 or 28 days after the establishment of MI. Subsequent analysis of the protein expression levels of the apoptosis proteins mentioned above demonstrated a blunted response, and the anti-apoptosis protein bcl-2 demonstrated an intense response following miR-17-5p silencing compared to the control group at 7 or 28 days. This anti-apoptosis effect of miR-17-5p silencing was accompanied by increased mRNA expression levels of ERK and P-ERK, as observed by western blot.

\section{Effect of miR-17-5p on histology of rat heart after MI}

After 7- or 28-day MI, as shown in Fig. 6, HE staining showed that infarction areas were decreased by 43.7 and $73.2 \%$ in the Ad-Antagomir-17 group and Ad-NC-Antagomir-17 group, respectively $(p<0.05)$. Masson staining also indicated that areas of collagen fibers were reduced by 53.3 and $61.1 \%$ after miR-17-5p silencing in the Ad-Antagomir-17 group and Ad-NC-Antagomir-17 group, respectively $(p<0.05)$. Therefore, downregulation of miR-17-5p could protect cardiac structure of rat heart after AMI.

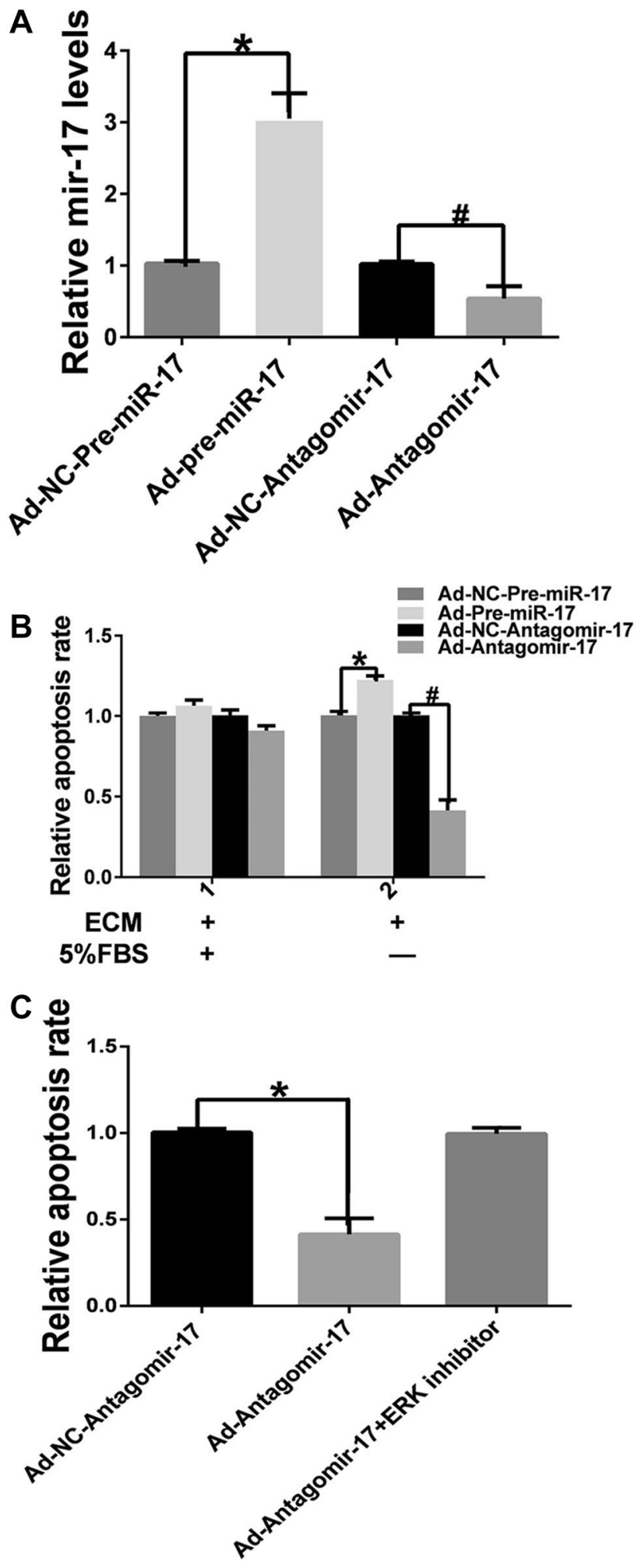

Effect of miR-17-5p on immunohistochemistry of rat heart after MI

After 7- or 28-day MI, as shown in Fig. 7, apoptosis of cardiac tissue cells was examined by TUNEL staining, which 
Fig. 2 Effects of miR-17-5p on the ERK pathway. a HUVECs were infected with adenovirus for $4 \mathrm{~h}$ and cultivated with ECM containing $5 \%$ FBS for $72 \mathrm{~h}$. Then, they were cultivated with ECM without FBS for another $24 \mathrm{~h}$ to induce apoptosis. The cells were collected, and the protein levels of ERK and P-ERK were measured. b Protein levels of ERK, P-ERK in the HUVECs mentioned above were examined by western blot, and ERK activity was evaluated by calculating P-ERK/ ERK. $\mathbf{c}$ mRNA levels of ERK were examined by PCR $(* p<0.05$ vs. Ad-NC-Pre-miR-17, ${ }^{*} p<0.05$ vs. Ad-NC-Antagomir-17, $n=3$ )

showed that downregulation of miR-17-5p inhibited the apoptosis of cardiac tissue cells compared to the Ad-NCAntagomir-17 group $(0.23 \pm 0.101$ vs. $0.61 \pm 0.056$ at 7 days after MI, $0.20 \pm 0.0108$ vs. $0.41 \pm 0.038$ at 28 days after I, $p<0.05)$. CD31, a marker of microcirculation formation, was stained and also indicated that miR-17-5p silencing promotes endothelial growth after MI compared to the AdNC-Antagomir- 17 group $(0.814 \pm 0.047$ vs. $0.360 \pm 0.166$ at 7 days after MI, $0.736 \pm 0.069$ vs. $0.432 \pm 0.181$ at 28 days after MI, $p<0.05)$. Thus, miR-17 silence could promote microcirculation of rat heart after AMI.

\section{Effect of miR-17-5p on rat heart function after MI}

At 7 or 28 days after MI, cardiac function was evaluated morphologically and quantitatively. Many index were used to determine the cardiac function, like ejection fractions (EF), fractional shortening (FS), end diastolic volume (EDV), End systolic volume (ESV), left ventricular internal dimension diastole (LVIDD), left ventricular internal dimension systole (LVIDS). As shown in Table 1, echocardiography revealed that downregulation of miR-17-5p enhanced heart function. The data mean percent decrease compared with heart function before AMI.

Heart function was evaluated by echocardiography before the models were built and at 7 and 28 days later. The percentages of heart function decrease in each group were calculated $(* p<0.05$ vs. Ad-NC-Antagomir-17).

\section{Discussion}

Many miRNAs have been reported to regulate senescence positively or negatively, but most studies have focused on the effect of miRNAs on the senescence of tumor cells at cellular level, or investigated the role of miRNAs in lower organisms (Du et al. 2015). Uniquely, our study systematically describes the functions of one specific miRNA, miR17. MicroRNA-17 has been investigated in many fields, such as B-cell lymphoma (Lu et al. 2011), acute organ-specific autoimmune disease (Battistella et al. 2015), glucocorticoid-induced osteoclast differentiation and function (de Kouchkovsky et al. 2013), and so on. It is involved in angiopoiesis (Shi et al. 2014), apoptosis (Shan et al. 2013), and
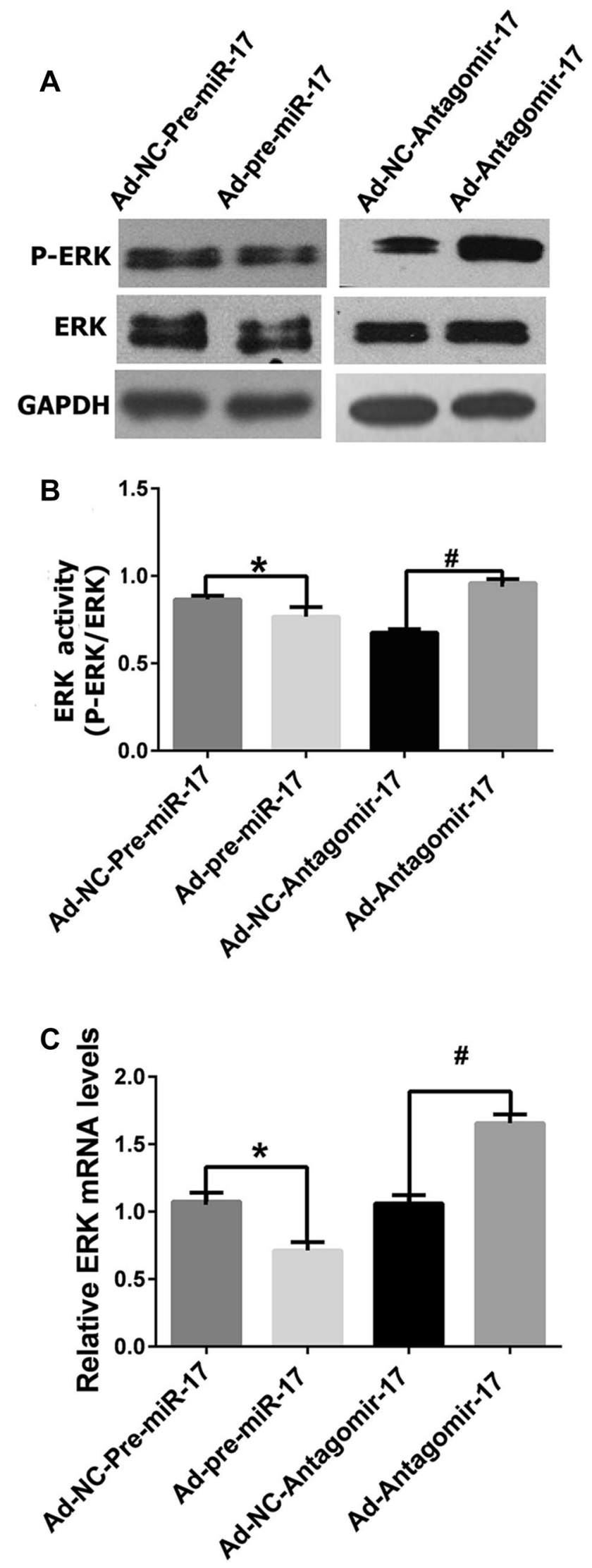
Fig. 3 Effect of miR-17-5p on apoptosis-related protein levels. a HUVECs were infected with adenovirus for $4 \mathrm{~h}$ and cultivated with ECM containing 5\% FBS for $72 \mathrm{~h}$. Then, they were cultivated with ECM without FBS for another $24 \mathrm{~h}$ to induce apoptosis. The cells were collected, and the protein levels of apoptosis-related protein were measured. b Protein levels of anti-apoptosis protein bcl-2 were measured. c Levels of apoptosis protein Bax was measured. d Relative protein levels of apoptosis protein caspas3. e Relative protein levels of apoptosis protein caspas 9 (* $p<0.05$ vs. Ad-NC-PremiR-17, ${ }^{\#} p<0.05$ vs. Ad-NCAntagomir-17, $n=3$ )
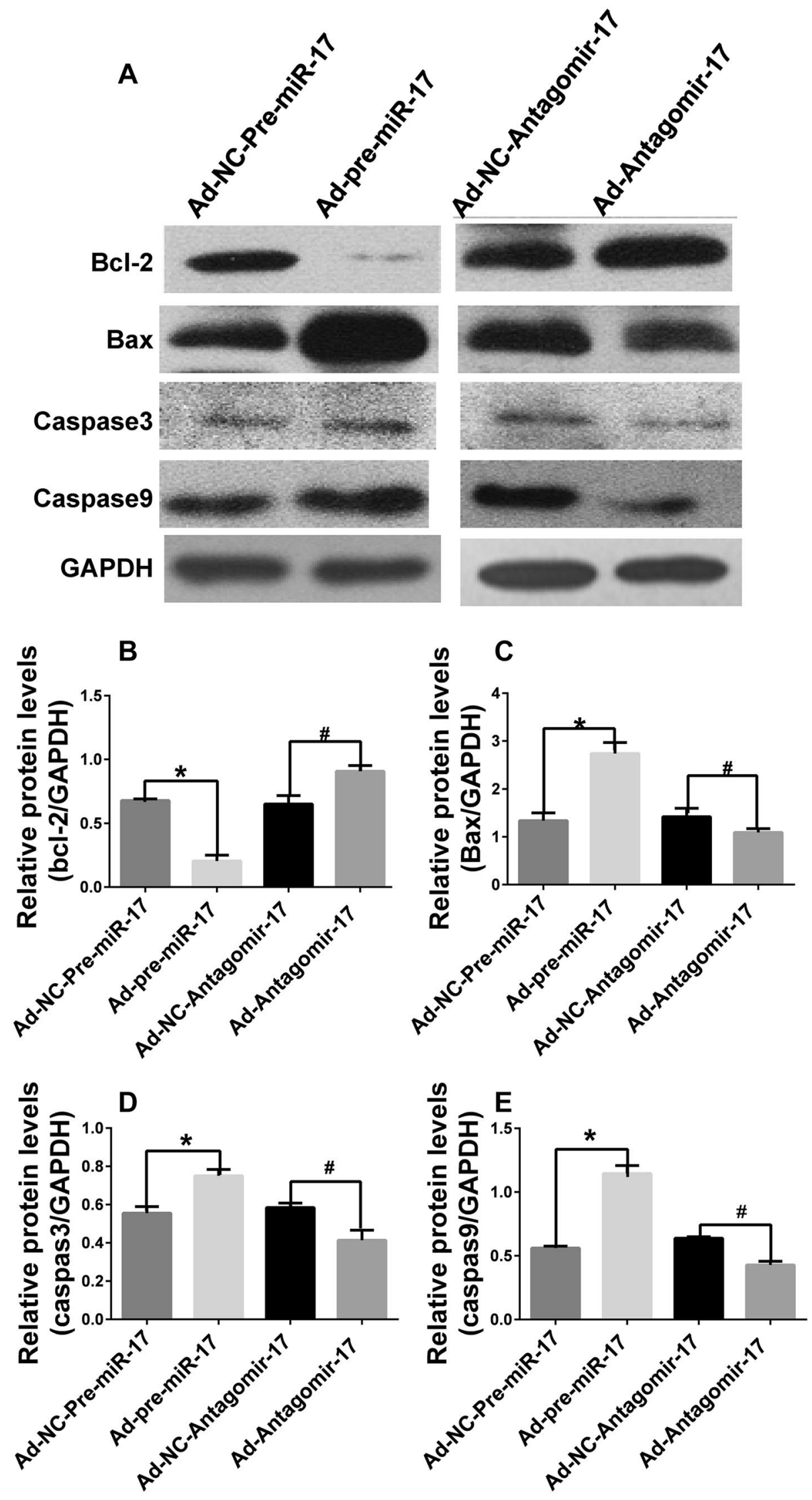
Fig. 4 Effects of miR-17-5p inhibition on the ERK pathway in injured cardiac tissue. After 7 or 28 days AMI, a mRNA expression of miR-17 was analyzed by real-time RT-PCR $(n=3$, each group contains 5 hearts). $\mathbf{b}$ mRNA expression of ERK was analyzed by real-time RT-PCR ( $n=3$, each group contains 5 hearts). c Protein expression of ERK, P-ERK were examined by western blot. d ERK protein activity was evaluated by P-ERK/ERK $(n=3$, each group contains 5 hearts) $(* p<0.05$ vs. Ad-NCAntagomir-17, ${ }^{\#} p<0.05$ vs. Ad-NC-Antagomir-17, $n=3$ )
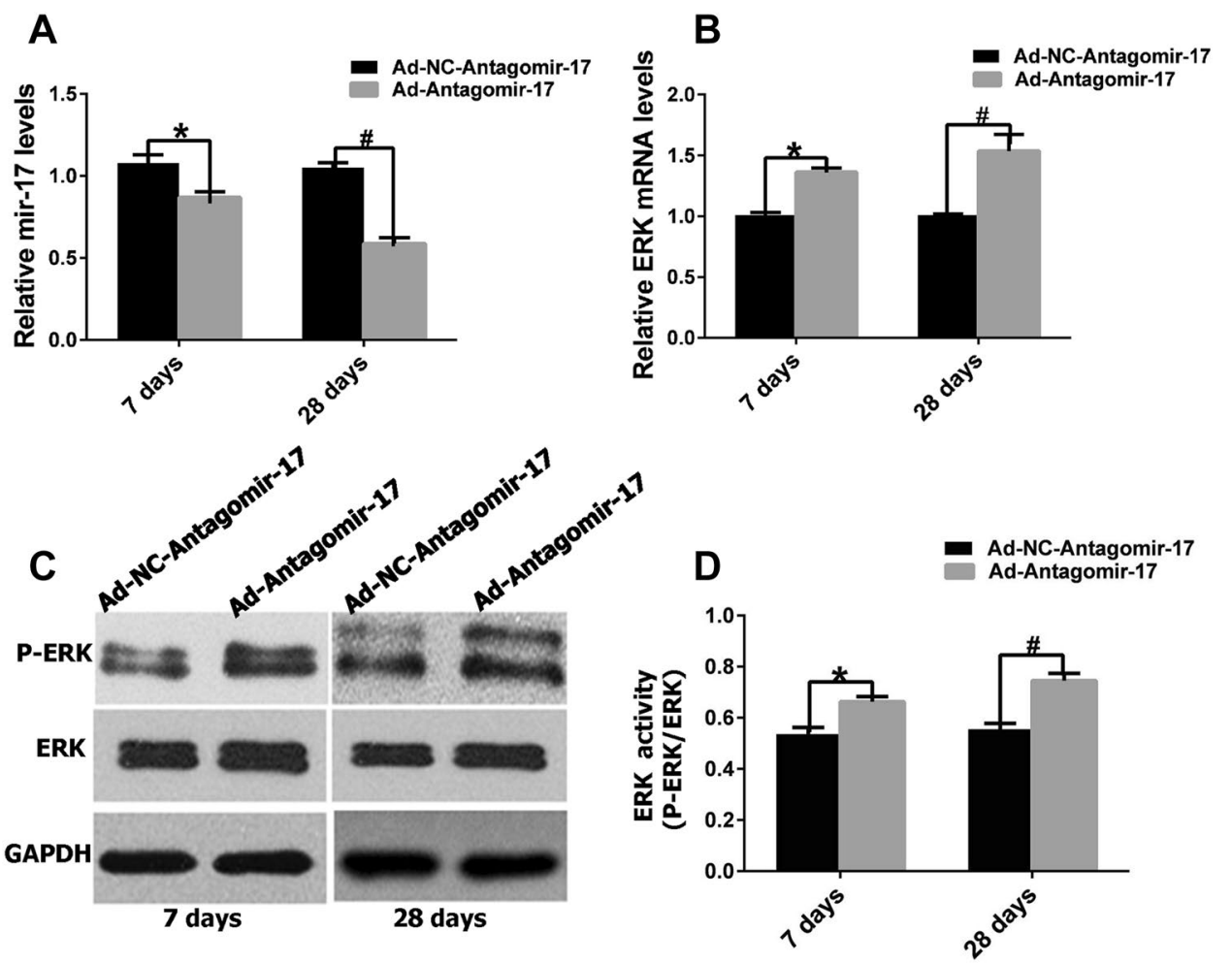

proliferation (Honegger et al. 2015) in tumor tissues. However, the relationship between miR-17 and cardiovascular disease and the mechanisms underlying that relationship are still unknown. It is reported that miR-17 is sufficient to induce cardiomyocyte proliferation, but have nothing to do with cardiomyocyte apoptosis (Chen et al. 2013). However, this study focused on the effect of miR-17 on the apoptosis of ECs induced by serum deprivation. Our results indicated that miR-17-5p silencing had a greater apoptosis inhibition effect on ECs under serum-deprived conditions and had a nearly insignificant effect on ECs under normal conditions. The apoptosis rate is low under normal circumstances; thus, the apoptosis mechanism could not be triggered in the cells grown under normal conditions. These results show that miR-17-5p plays an important role in making life or death choices in ECs when apoptosis is triggered.

One major question being investigated in this study was how miR-17-5p silencing decreases the apoptotic rate of ECs in response to serum deprivation. Apoptosis can be triggered by extracellular stimuli via the death receptor and mitochondrial-mediated pathways (Scarabelli et al. 2002). The ERK pathway plays an important role in several cellular processes, such as cell survival, cell proliferation and apoptosis. It has been reported that activated ERK is responsible for high levels of cell growth in various cancers (Dhillon et al. 2007; Yang et al. 2013). Meanwhile, inhibition of activated ERK is associated with apoptosis (Salminen et al. 2010). Inhibition of miR-17-5p promotes the expression of activated
ERK both in vivo and in vitro. Thus, we hypothesized that downregulation of miR-17-5p would decrease the apoptotic rate of endothelial cells both in vivo and in vitro. Proteins of the bcl-2 family play a pivotal role in apoptosis (Baell and Huang 2002; Goodsell 2002). BCL-2 interacting mediator of cell death (BIM) can be phosphorylated by members of mitogen activated protein kinase family. Extracellular signalregulated kinase 1/2 (ERK1/2)-mediated phosphorylation stimulates BIM degradation via the proteasome (Luciano et al. 2003; Kennedy et al. 2017). Bcl-2 is thought to be an anti-apoptotic protein and can inhibit the activity of the caspase cascade after it is initiated by the mitochondrial release of cytochrome c (Rosse et al. 1998; Cao et al. 2015). In contrast, bax is thought to be a proapoptotic protein of the bcl-2 family (Sedlak et al. 1995; Kluck et al. 1997), which can result in permeabilization of mitochondria (Rosse et al. 1998; Cao et al. 2015). We also detected the protein levels of caspase 3 and caspase 9, which, when in their active state, play a crucial role in the final step of apoptosis (Shebaby et al. 2014). Our research found that downregulation of miR17 increased the protein level of bcl-2, while levels of the bax/caspase 3/caspase 9 proteins were decreased, which is consistent with inhibition of apoptosis.

Heart failure after myocardial infarction is a clinically common phenomenon. There is high morbidity and mortality following myocardial infarction, because the remaining heart tissue has to maintain adequate cardiac output, similar to the current management of congestive 
Fig. 5 Effects of miR-17-5p inhibition on apoptosis-related proteins in injured cardiac tissue. After 7 or 28 days AMI, a Western blot was used to detected apoptosis-related proteins. b Protein expression of anti-apoptosis protein bcl-2 was examined by western blot. c Levels of apoptosis protein Bax was measured by western blot. d Protein expression of apoptosis protein caspas 3 was examined by western blot. e Relative protein levels of apoptosis protein caspas9 $(n=3$, each group contains 5 hearts) $(* p<0.05$ vs. Ad-NC-Antagomir-17, ${ }^{\#} p<0.05$ vs. Ad-NC-Antagomir-17, $n=3$ )
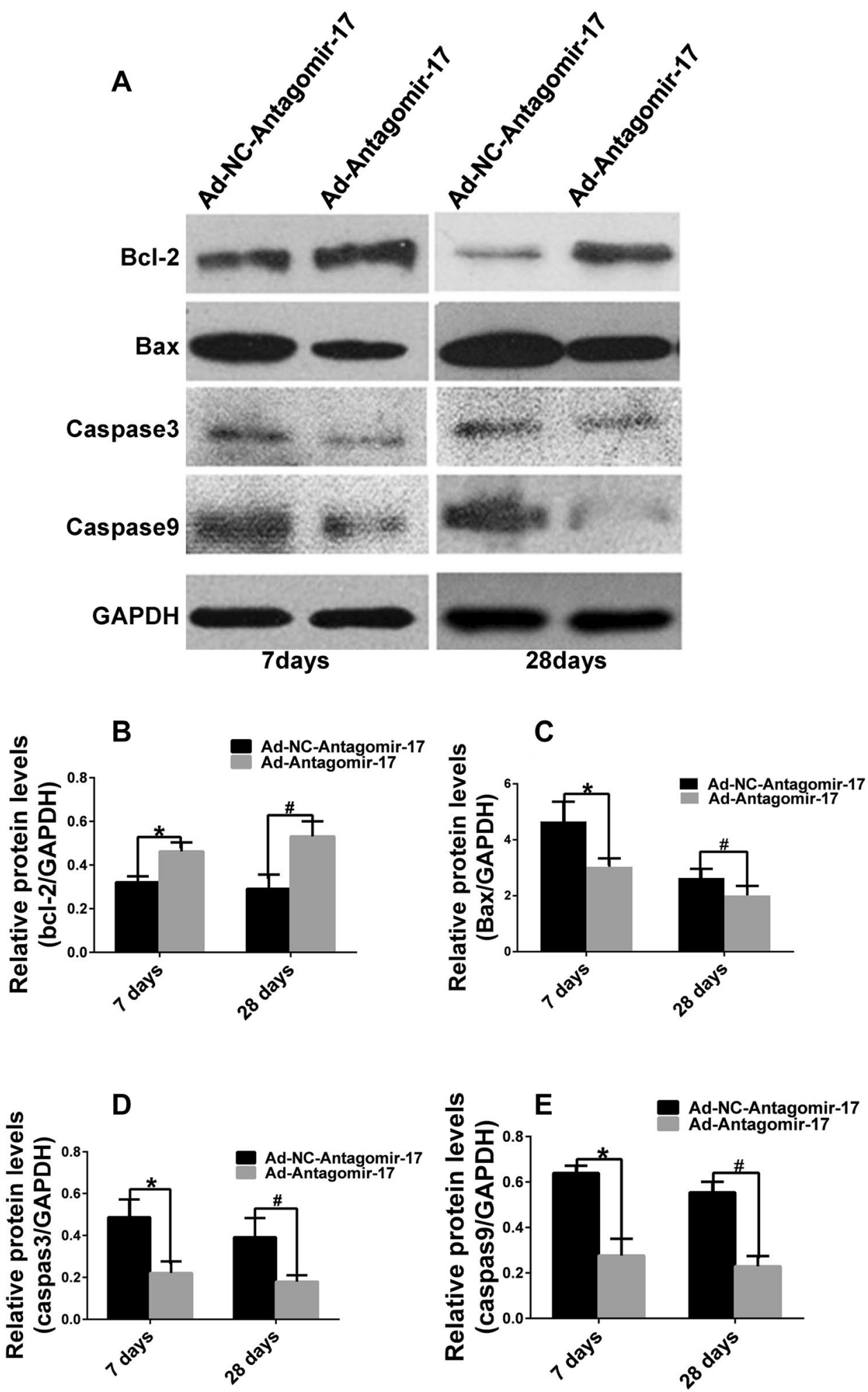

heart failure (Kanashiro-Takeuchi et al. 2015). Our data showed that have shown that heart failure after myocardial infarction is closely related to the microcirculation damage of the heart (Teunissen et al. 2015). Heart function can be protected if microcirculation is improved after myocardial infarction, which would prevent symptoms of heart failure. Research has verified that inhibiting the expression of miR-17-5p can prevent endothelial cells from undergoing apoptosis and can also promote tube formation by vessel endothelial cells. Therefore, 
Fig. 6 Effect of miR-17-5p inhibition on the histopathology in heart tissue after myocardial infarction at 7 or 28 days after the induction of AMI. a, b Representative hematoxylin and eosin-stained cardiac tissues from each experimental group ( $n=5$, light microscope, $\times 400)$. C, d Representative Masson trichrome-stained heart tissues. Collagen is stained blue ( $n=5$, light microscope, $\times 200)\left({ }^{\#} p<0.05\right.$ vs. *Ad-NCAntagomir-17)
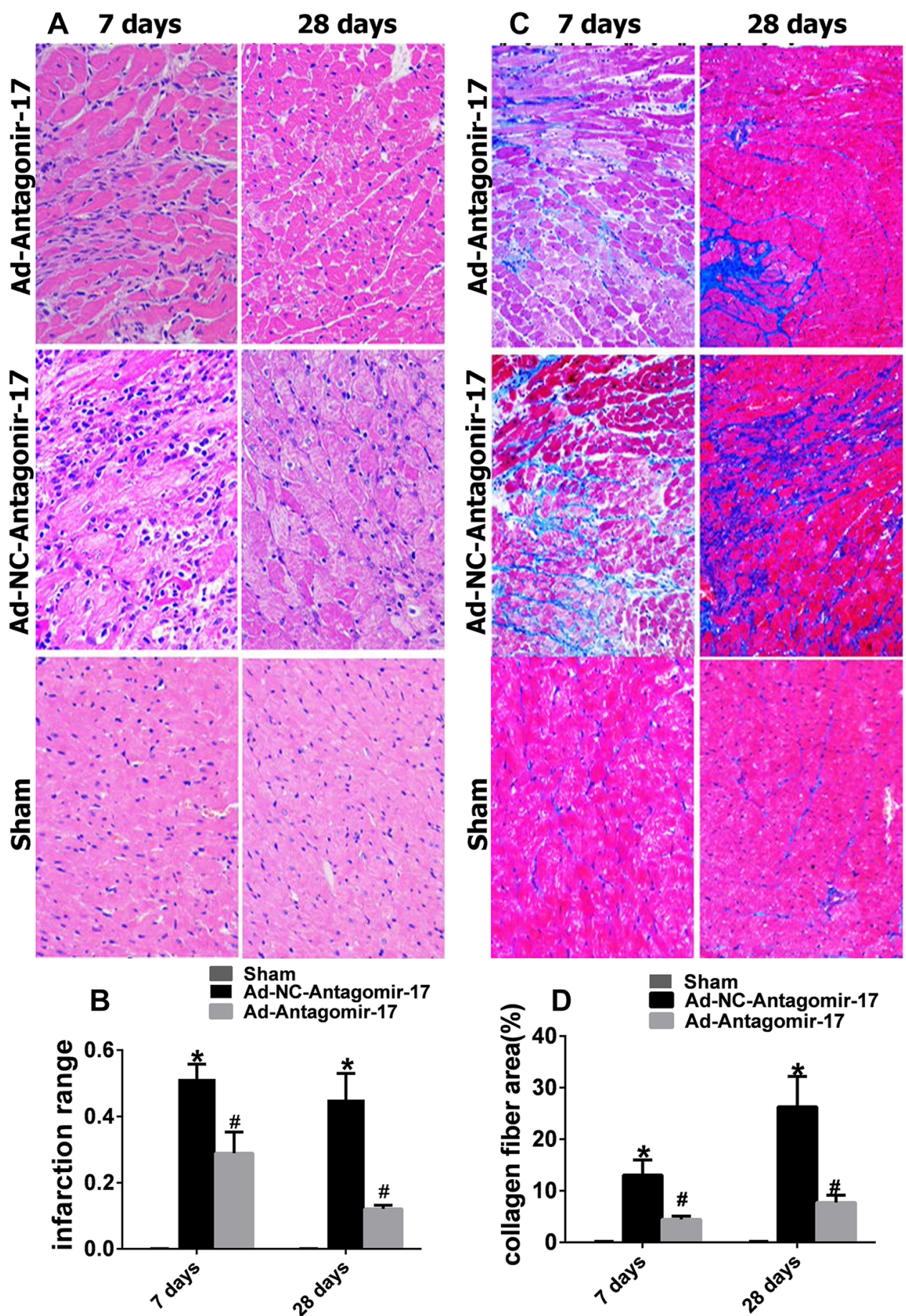

MiR-17-5p silencing protects heart function after AMI by improving the microcirculation of the heart tissue, which is through decreasing the rate of apoptosis and repairing vascular injury. The effect of miR-17 on the heart is still not entirely clear, and many more potential mechanisms underlying this effect must be investigated.

\section{Conclusion}

Collectively, this study provided novel insights into the mechanism of miR-17-5p regulating endothelium apoptosis. Actually, apoptosis was inhibited by the 
Fig. 7 Effect of miR-17-5p inhibition on the histopathology in heart tissue after myocardial infarction at 7 or 28 days after the induction of AMI. a, b Representative cardiac sections with immunohistochemical staining for CD31. Black arrows indicate areas of endothelial recovery, which are stained dark brown $(n=5$, light microscope, $\times 400)$. c, d Representative TUNELstained cardiac sections. Black arrows indicate positive cells, which are stained dark brown $(n=5$, light microscope, $\times 400)$ $\left({ }^{\#} p<0.05\right.$ vs. *Ad-NC-Antagomir-17)
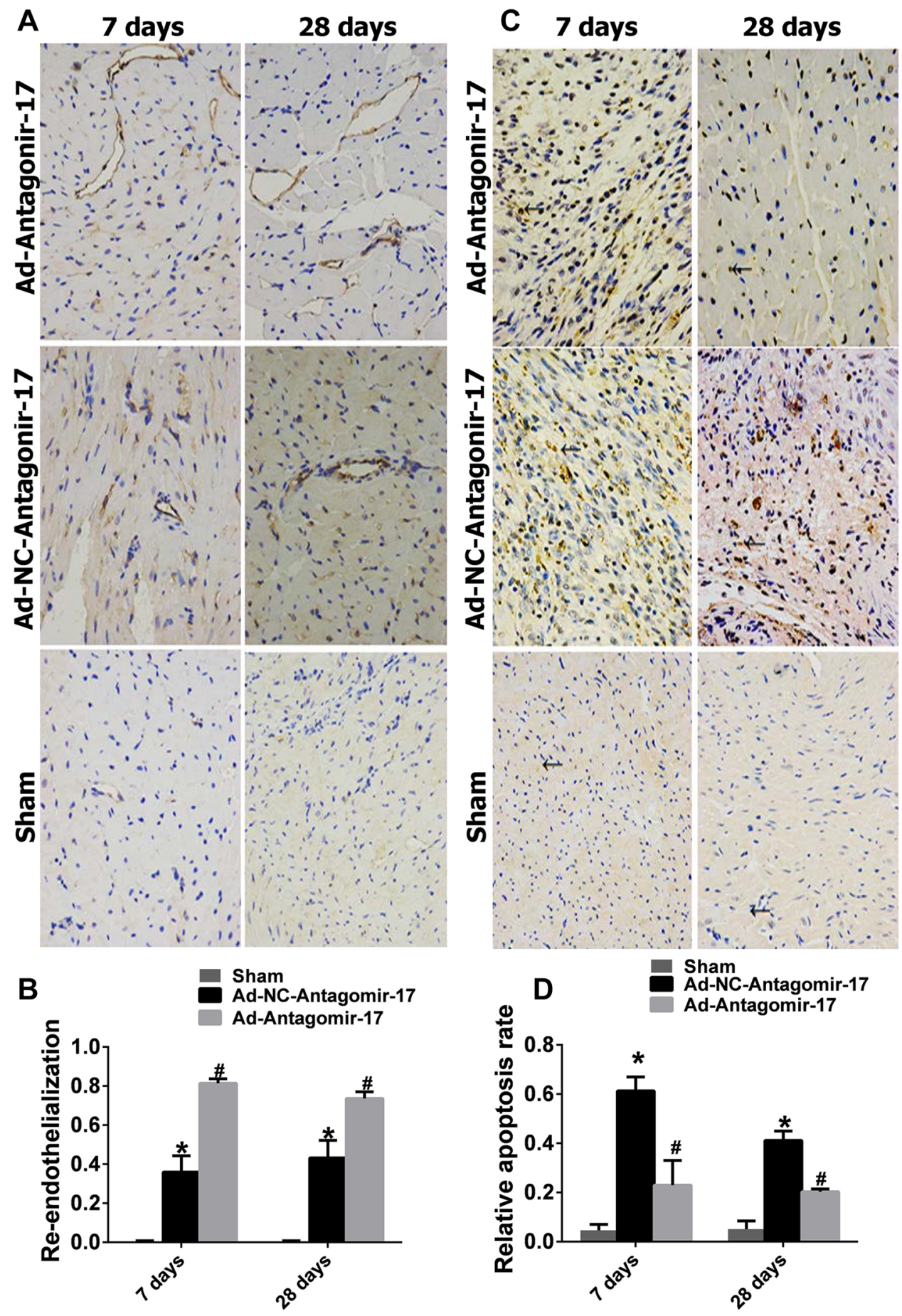

downregulation of miR-17-5p through activation of the ERK pathway. MiR-17-5p silencing protects heart function after AMI by improving the microcirculation of the heart tissue by decreasing the rate of apoptosis and repairing vascular injury. 
Table 1 Effect of miR-17-5p inhibition on heart function after myocardial infarction

\begin{tabular}{lcccc}
\hline & $\begin{array}{l}\text { Ad-NC-Antagomir-17 } \\
28 \text { days }\end{array}$ & $\begin{array}{l}\text { Ad-Antagomir-17 } \\
28 \text { days }\end{array}$ & $\begin{array}{l}\text { Ad-NC-Antagomir-17 } \\
7 \text { days }\end{array}$ & $\begin{array}{l}\text { Ad-Antagomir-17 } \\
7 \text { days }\end{array}$ \\
\hline EF & $0.3723 \pm 0.05393$ & $0.06021 \pm 0.02443^{*}$ & $0.3406 \pm 0.03382$ & $0.08890 \pm 0.02123^{*}$ \\
FS & $-0.5453 \pm 0.1118$ & $-0.1298 \pm 0.05233^{*}$ & $-0.5048 \pm 0.07902$ & $-0.1706 \pm 0.04511^{*}$ \\
EDV & $2.879 \pm 0.1945$ & $1.022 \pm 0.2184^{*}$ & $2.016 \pm 0.3738$ & $0.3111 \pm 0.05300^{*}$ \\
ESV & $28.00 \pm 3.372$ & $6.000 \pm 2.517^{*}$ & $24.33 \pm 3.528$ & $8.444 \pm 2.764^{*}$ \\
LVIDD & $0.9624 \pm 0.2147$ & $0.2828 \pm 0.05307^{*}$ & $0.8422 \pm 0.1362$ & $0.2681 \pm 0.1519^{*}$ \\
LVIDS & $1.274 \pm 0.2665$ & $0.4556 \pm 0.1056^{*}$ & $2.924 \pm 0.5913$ & $0.8479 \pm 0.3661^{*}$ \\
\hline
\end{tabular}

${ }^{*} P<0.05$ which represent that the indicators (EF, FS, EDV, ESV, LVIDD and LVIDS) had significant differences between downregulation group (Ad-Aatagomir-17) and control group (Ad-NC-Aatagomir-17)

\begin{abstract}
Acknowledgements This work was supported by award from the National Natural Science Foundation of China to Jing Chen (No. 81200156), Tao Fan (No. 81700093) and Hong Jiang (No. 81170195).
\end{abstract}

Open Access This article is distributed under the terms of the Creative Commons Attribution 4.0 International License (http://creativeco mmons.org/licenses/by/4.0/), which permits unrestricted use, distribution, and reproduction in any medium, provided you give appropriate credit to the original author(s) and the source, provide a link to the Creative Commons license, and indicate if changes were made.

\section{References}

Al Suwaidi J, Berger PB, Holmes DR Jr (2000) Coronary artery stents. JAMA 284:1828-1836

Baell JB, Huang DC (2002) Prospects for targeting the Bcl-2 family of proteins to develop novel cytotoxic drugs. Biochem Pharmacol 64:851-863

Battistella M, Romero M, Castro-Vega LJ, Gapihan G, Bouhidel F, Bagot M, Feugeas JP, Janin A (2015) The high expression of the microRNA 17-92 cluster and its paralogs, and the downregulation of the target gene PTEN, is associated with primary cutaneous B-cell lymphoma progression. J Invest Dermatol 135:1659-1667

Bonauer A, Carmona G, Iwasaki M, Mione M, Koyanagi M, Fischer A, Burchfield J, Fox H, Doebele C, Ohtani K, Chavakis E, Potente M, Tjwa M, Urbich C, Zeiher AM, Dimmeler S (2009) MicroRNA92 a controls angiogenesis and functional recovery of ischemic tissues in mice. Science 324:1710-1713

Cao ZH, Zheng QY, Li GQ, Hu XB, Feng SL, Xu GL, Zhang KQ (2015) STAT1-mediated down-regulation of Bcl-2 expression is involved in IFN-gamma/TNF-alpha-induced apoptosis in NIT-1 cells. PLoS One 10:e0120921

Chen J, Huang ZP, Seok HY, Ding J, Kataoka M, Zhang Z, Hu X, Wang G, Lin Z, Wang S, Pu WT, Liao R, Wang DZ (2013) mir-17-92 cluster is required for and sufficient to induce cardiomyocyte proliferation in postnatal and adult hearts. Circ Res 112:1557-1566

Dangas GD, Claessen BE, Caixeta A, Sanidas EA, Mintz GS, Mehran $\mathrm{R}$ (2010) In-stent restenosis in the drug-eluting stent era. J Am Coll Cardiol 56:1897-1907

de Kouchkovsky D, Esensten JH, Rosenthal WL, Morar MM, Bluestone JA, Jeker LT (2013) microRNA-17-92 regulates IL-10 production by regulatory $\mathrm{T}$ cells and control of experimental autoimmune encephalomyelitis. J Immunol 191:1594-1605

Dhillon AS, Hagan S, Rath O, Kolch W (2007) MAP kinase signalling pathways in cancer. Oncogene 26:3279-3290
Doebele C, Bonauer A, Fischer A, Scholz A, Reiss Y, Urbich C, Hofmann WK, Zeiher AM, Dimmeler S (2010) Members of the microRNA-17-92 cluster exhibit a cell-intrinsic antiangiogenic function in endothelial cells. Blood 115:4944-4950

Du W, Pan Z, Chen X, Wang L, Zhang Y, Li S, Liang H, Xu C, Zhang Y, Wu Y, Shan H, Lu Y (2014) By targeting Stat3 microRNA$17-5 p$ promotes cardiomyocyte apoptosis in response to ischemia followed by reperfusion. Cell Physiol Biochem 34:955-965

Du WW, Li X, Li T, Li H, Khorshidi A, Liu F, Yang BB (2015) The microRNA miR-17-3p inhibits mouse cardiac fibroblast senescence by targeting Par4. J Cell Sci 128:293-304

Farb A, Boam AB (2007) Stent thrombosis redux - the FDA perspective. N Engl J Med 356:984-987

Finn AV, Joner M, Nakazawa G, Kolodgie F, Newell J, John MC, Gold HK, Virmani R (2007) Pathological correlates of late drug-eluting stent thrombosis: strut coverage as a marker of endothelialization. Circulation 115:2435-2441

Goodsell DS (2002) The molecular perspective: Bcl-2 and apoptosis. Oncologist 7:259-260

Honegger A, Schilling D, Bastian S, Sponagel J, Kuryshev V, Sultmann H, Scheffner M, Hoppe-Seyler K, Hoppe-Seyler F (2015) Dependence of intracellular and exosomal microRNAs on viral E6/E7 oncogene expression in HPV-positive tumor cells. PLoS Pathog 11:e1004712

Inoue T, Croce K, Morooka T, Sakuma M, Node K, Simon DI (2011) Vascular inflammation and repair: implications for re-endothelialization, restenosis, and stent thrombosis. JACC Cardiovasc Interv 4:1057-1066

Ji R, Cheng Y, Yue J, Yang J, Liu X, Chen H, Dean DB, Zhang C (2007) MicroRNA expression signature and antisense-mediated depletion reveal an essential role of microRNA in vascular neointimal lesion formation. Circ Res 100:1579-1588

Joner M, Finn AV, Farb A, Mont EK, Kolodgie FD, Ladich E, Kutys R, Skorija K, Gold HK, Virmani R (2006) Pathology of drug-eluting stents in humans: delayed healing and late thrombotic risk. J Am Coll Cardiol 48:193-202

Kanashiro-Takeuchi RM, Szalontay L, Schally AV, Takeuchi LM, Popovics P, Jaszberenyi M, Vidaurre I, Zarandi M, Cai RZ, Block NL, Hare JM, Rick FG (2015) New therapeutic approach to heart failure due to myocardial infarction based on targeting growth hormone-releasing hormone receptor. Oncotarget 6:9728-9739

Kennedy D, Mnich K, Oommen D, Chakravarthy R, Almeida-Souza L, Krols M, Saveljeva S, Doyle K, Gupta S, Timmerman V, Janssens S, Gorman AM, Samali A (2017) HSPB1 facilitates ERKmediated phosphorylation and degradation of BIM to attenuate endoplasmic reticulum stress-induced apoptosis. Cell Death Dis 8:e3026-

Kluck RM, Bossy-Wetzel E, Green DR, Newmeyer DD (1997) The release of cytochrome $\mathrm{c}$ from mitochondria: a primary site for Bcl-2 regulation of apoptosis. Science 275:1132-1136 
Krol J, Loedige I, Filipowicz W (2010) The widespread regulation of microRNA biogenesis, function and decay. Nat Rev Genet 11:597-610

Lu Y, Qin W, Shen T, Dou L, Man Y, Wang S, Xiao C, Li J (2011) The antioxidant $\mathrm{N}$-acetylcysteine promotes atherosclerotic plaque stabilization through suppression of RAGE, MMPs and NF-kappaB in ApoE-deficient mice. J Atheroscler Thromb 18:998-1008

Luciano F, Jacquel A, Colosetti P, Herrant M, Cagnol S, Pages G, Auberger P (2003) Phosphorylation of Bim-EL by Erk1/2 on serine 69 promotes its degradation via the proteasome pathway and regulates its proapoptotic function. Oncogene 22:6785-6793

Norbury CJ (2010) 3' Uridylation and the regulation of RNA function in the cytoplasm. Biochem Soc Trans 38:1150-1153

Pan Z, Sun X, Ren J, Li X, Gao X, Lu C, Zhang Y, Sun H, Wang Y, Wang H, Wang J, Xie L, Lu Y, Yang B (2012) miR-1 exacerbates cardiac ischemia-reperfusion injury in mouse models. PLoS One 7:e50515

Ren XP, Wu J, Wang X, Sartor MA, Qian J, Jones K, Nicolaou P, Pritchard TJ, Fan GC (2009) MicroRNA-320 is involved in the regulation of cardiac ischemia/reperfusion injury by targeting heat-shock protein 20. Circulation 119:2357-2366

Rosse T, Olivier R, Monney L, Rager M, Conus S, Fellay I, Jansen B, Borner C (1998) Bcl-2 prolongs cell survival after Bax-induced release of cytochrome c. Nature 391:496-499

Salminen A, Lehtonen M, Paimela T, Kaarniranta K (2010) Celastrol: molecular targets of thunder god vine. Biochem Biophys Res Commun 394:439-442

Scarabelli TM, Stephanou A, Pasini E, Comini L, Raddino R, Knight RA, Latchman DS (2002) Different signaling pathways induce apoptosis in endothelial cells and cardiac myocytes during ischemia/reperfusion injury. Circ Res 90:745-748

Sedlak TW, Oltvai ZN, Yang E, Wang K, Boise LH, Thompson CB, Korsmeyer SJ (1995) Multiple Bcl-2 family members demonstrate selective dimerizations with Bax. Proc Natl Acad Sci USA 92:7834-7838

Shan SW, Fang L, Shatseva T, Rutnam ZJ, Yang X, Du W, Lu WY, Xuan JW, Deng Z, Yang BB (2013) Mature miR-17-5p and passenger miR-17-3p induce hepatocellular carcinoma by targeting PTEN, GalNT7 and vimentin in different signal pathways. J Cell Sci 126:1517-1530

Shebaby WN, Mroueh M, Bodman-Smith K, Mansour A, Taleb RI, Daher CF, El-Sibai M (2014) Daucus carota pentane-based fractions arrest the cell cycle and increase apoptosis in MDA-MB-231 breast cancer cells. BMC Complement Altern Med 14:387

Shi C, Qi J, Huang P, Jiang M, Zhou Q, Zhou H, Kang H, Qian N, Yang Q, Guo L, Deng L (2014) MicroRNA-17/20a inhibits glucocorticoid-induced osteoclast differentiation and function through targeting RANKL expression in osteoblast cells. Bone 68:67-75

Teunissen PF, de Waard GA, Hollander MR, Robbers LF, Danad I, Biesbroek PS, Amier RP, Echavarria-Pinto M, Quiros A, Broyd C, Heymans MW, Nijveldt R, Lammertsma AA, Raijmakers PG, Allaart CP, Lemkes JS, Appelman YE, Marques KM, Bronzwaer JG, Horrevoets AJ, van Rossum AC, Escaned J, Beek AM, Knaapen P, van Royen N (2015) Doppler-derived intracoronary physiology indices predict the occurrence of microvascular injury and microvascular perfusion deficits after angiographically successful primary percutaneous coronary intervention. Circ Cardiovasc Interv 8:e001786

van Rooij E (2011) The art of microRNA research. Circ Res 108:219-234

Wang X, Zhang X, Ren XP, Chen J, Liu H, Yang J, Medvedovic M, Hu Z, Fan GC (2010) MicroRNA-494 targeting both proapoptotic and antiapoptotic proteins protects against ischemia/reperfusioninduced cardiac injury. Circulation 122:1308-1318

Yang SH, Sharrocks AD, Whitmarsh AJ (2013) MAP kinase signalling cascades and transcriptional regulation. Gene 513:1-13

Yu Z, Willmarth NE, Zhou J, Katiyar S, Wang M, Liu Y, McCue PA, Quong AA, Lisanti MP, Pestell RG (2010) microRNA 17/20 inhibits cellular invasion and tumor metastasis in breast cancer by heterotypic signaling. Proc Natl Acad Sci USA 107:8231-8236

Zhang Y, Li W, Ou L, Wang W, Delyagina E, Lux C, Sorg H, Riehemann K, Steinhoff G, Ma N (2012) Targeted delivery of human VEGF gene via complexes of magnetic nanoparticle-adenoviral vectors enhanced cardiac regeneration. PLoS One 7:e39490 